\title{
Vibrational analysis of extracellular matrix scaffolds: comparison of skin, dermis, cartilage and subchondral bone using oct and vibrational analysis
}

\begin{abstract}
For four decades clinicians have used scaffolds composed of extracellular matrices (ECMs) as replacements for a variety of tissues. These scaffolds contain collagen from different subfamilies and to a first approximation mimic the structure of the host tissue. To avoid problems such as intimal hyperplasia and stress-shielding it is important that the mechanical properties of the host and implant materials be matched.

We have reported that the use of optical cohesion tomography (OCT) in concert with vibrational analysis is a useful tool to evaluate mechanical properties of scaffolds to replace extracellular matrices in animals and humans as well to study the onset and pathogenesis of diseases. In this paper, we compare the mechanical properties of a number of ECMs to establish the mechanical requirements for scaffolding materials. The results presented in this paper suggest that the moduli of elastic tissue, fibrous collagen and subchondral bone are characteristic finger prints of structural components of ECMs that can be non-invasively and non-destructive measured using $\mathrm{OCT}$ and vibrational analysis.
\end{abstract}

Keywords: mechanical properties, modulus, tissue, optical coherence tomography, vibrational analysis, skin, dermis, scar, cartilage, subchondral bone, collagen, extracellular matrix
Volume 2 Issue 6 - 2017

\author{
Ruchit G Shah,' Frederick H Silver ${ }^{2}$ \\ 'Graduate Program in Biomedical Engineering, Rutgers, The \\ State University of New Jersey, USA \\ ${ }^{2}$ Department of Pathology and Laboratory Medicine, Rutgers, \\ The State University of New Jersey, USA
}

\author{
Correspondence: Frederick H Silver, Department of \\ Pathology and Laboratory Medicine, Robert Wood Johnson \\ Medical School, Rutgers, The State University of New Jersey, 675 \\ Hoes Lane, Piscataway, NJ 08854, USA, \\ Email fhsilver@hotmail.com
}

Received: June II, 2017| Published: September 06, 2017

\section{Introduction}

Collagenous scaffolds are used both as decellularized and/or cellseeded substrates for tissue repair and regeneration. ${ }^{1}$ One of the major problems with tissue repair and regeneration is selecting a scaffold with similar mechanical properties to that of the host tissue; it has been reported that mechanical mismatches between implant and host tissues result in stress concentrations and cellular hyperplasia at the interface. ${ }^{2}$ In small diameter vascular grafts, this can lead to intimal hyperplasia and graft stenosis and has been a difficult problem to overcome. For this reason, it is important to match the mechanical properties of host tissue with that of tissue engineered implants and grafts.

Several methods have been used to evaluate the mechanical properties of ECMs over the last 40 years including uniaxial and biaxial tensile testing, indentation and rotational tests. ${ }^{3,4}$ In addition, new tests including ultrasound elastography, optical cohesion tomography (OCT), magnetic resonance elastography, optical cohesion elastography, and vibrational analysis combined with OCT have been used to study the mechanical properties of tissues in health and disease. ${ }^{2}$ Most of these techniques require the assumptions that the material is linearly elastic, Poisson's ratio is close to 0.5 and that viscoelasticity does not dramatically affect the resulting properties. However, ECMs in general are non-linear materials that are viscoelastic and they exhibit an upward curvature to the stress-strain curve. These facts make determination of the modulus (stiffness) of scaffolds very difficult to measure since the tangent to the stress-strain curve is constantly changing. ${ }^{2,5-7}$ However, despite all of these problems, there is a need to be able to characterize the mechanical properties of scaffolds and ECMs since this would give clinicians valuable information concerning the mechanical properties of a wide variety of tissues that contain fibrous collagen in different physical forms. In addition, workers could evaluate quantitative changes associated with tumor formation, osteoarthritis, corneal diseases, wound healing, scarring and the efficacy of cosmetic and surgical treatments.

Previous published studies have reported methods to evaluate the viscoelastic mechanical properties of $\operatorname{skin}^{8}$ and decellularized dermis. ${ }^{5,69-11}$ Recently we have reported the use of optical coherence tomography (OCT) and vibrational analysis to non-invasively and non-destructively measure the mechanical properties of decellularized human dermis in vitro ${ }^{5,6}$ and human skin in vivo ${ }^{12}$ this technique measures the resonant frequency of a tissue or polymer and then converts the resonant frequency into a modulus. In addition, we have reported that moduli measurements made at the resonant frequency of ECMs and silicone rubber are quasi-elastic, since the viscous contribution to the mechanical behavior is as low as $2 \%$ to $4 \% .{ }^{11}$ Essentially the modulus measured at the resonant frequency is the elastic modulus of the material being studied. ${ }^{11}$ Measurements made at the resonant frequency therefore remove to a first approximation, the time-dependence of the material.

In this paper, we present data comparing the mechanical properties of pig and human skin, human scar tissue, decellularized human dermis, bovine cartilage and bovine bone. Our results suggest that using OCT and vibrational analysis the characteristic moduli of elastic fibers, collagen fibers and subchondral bone can be measured non- 
invasively and non-destructively. Scaffold characterization using measurements from OCT in combination with vibrational analysis provides additional information to use to evaluate tissue engineering products.

\section{Methods}

\section{Sample preparation}

Human decellularized dermis was obtained from allograft tissue as described previously ${ }^{5,6,-11}$ (Table 1). Decellularized human dermal samples were tested after immersion in phosphate buffer solution as described elsewhere. ${ }^{5,6}$ All samples were tested wet after soaking in phosphate buffer solution at $\mathrm{pH} 7.4$ for at least 30 minutes. Processing and testing steps were conducted at $22^{\circ} \mathrm{C}$.

Depilated pig skin, with a thickness of approximately $3 \mathrm{~mm}$, composed of epidermis and dermis, was obtained at slaughter from Spear Products (Coopersburg, PA) and stored at $4^{\circ} \mathrm{C}$. All samples were tested wet after soaking in phosphate buffer solution at $\mathrm{pH} 7.4$ for at least 30 minutes. Processing and sample testing steps were conducted at $22^{\circ} \mathrm{C}$ as described previously. 5,6

Table I Resonant frequencies and moduli values for ECM components measured using OCT and vibrational analysis

\begin{tabular}{lllll}
\hline Sample & Resonant frequency $(\mathbf{H z})$ & Vibrational testing modulus $(\mathbf{M P a})$ & Strain $(\%)$ & Thickness $(\mathbf{m m})$ \\
\hline Decellularized Dermis & $153.33 \pm 5.77$ & $2.57 \pm 0.2$ & 5 & 1.07 \\
& $246.67 \pm 5.77$ & $6.65 \pm 0.31$ & 14 & $\mathrm{NA}$ \\
& $346.67 \pm 5.77$ & $13.14 \pm 0.44$ & 20 & $\mathrm{NA}$ \\
Human skin & $73.33 \pm 5.77$ & $0.73 \pm 0.11$ & 2 & 3.05 \\
Scar tissue & $220 \pm 10$ & $4.2 \pm 0.38$ & 2 & 2.08 \\
Pig skin & $93.33 \pm 5.77$ & $0.77 \pm 0.094$ & 5 & \\
& $203.33 \pm 5.77$ & $3.61 \pm 0.2$ & 2 & \\
Bovine cartilage & $246.67 \pm 5.77$ & $4.96 \pm 0.23$ & & \\
& $550 \pm 10$ & $24.65 \pm 0.53$ & & 2 \\
Subchondral bone & $663.33 \pm 5.77$ & $35.03 \pm 0.61$ & $31.92 \pm 1.03$ & 29 \\
\hline
\end{tabular}

Bovine femoral cartilage and subchondral bone with a thickness of approximately $2 \mathrm{~mm}$ was obtained from Spear Products (Coopersburg, PA) and stored at $-40^{\circ} \mathrm{C}$ until it was tested. It was thawed before use and soaked in phosphate buffer at $\mathrm{pH} 7.4$ for at least 30 minutes before testing at $22^{\circ} \mathrm{C}$. Cartilage was removed from the subchondral bone by mechanical scraping using a surgical blade to evaluate the mechanical properties of the bone alone after testing both bone and cartilage together.

Human skin and normal scar tissue were evaluated in vivo to demonstrate the clinical use of the OCT and vibrational techniques. The scar tissue was the result of a small thermal burn wound smaller than the size of a dime that was depigmented after healing.

\section{Mechanical testing}

OCT and vibrational analysis In Vitro: Transverse forces were applied to the sample by positioning an acoustic loudspeaker (Intervox S225RA-40) beneath the sample. A function generator (Agilent) was used to drive the speaker with sinusoidal waveforms at varying amplitude and frequency as discussed previously. ${ }^{5,6}$

Transverse sample displacement was measured by spectraldomain optical coherence tomography (SD-OCT), a non-contact, interferometric technique as discussed previously., ${ }^{5,6}$ The SD-OCT system uses a fiber-coupled super luminescent diode light source with $1325 \mathrm{~nm}$ center wavelength and $100 \mathrm{~nm}$ bandwidth (full-width at half maximum) as described previously.,

The resonant frequency of each sample was initially estimated by measuring the transverse displacement resulting from sinusoidal driving frequencies ranging from $50 \mathrm{~Hz}$ to $1000 \mathrm{~Hz}$, in steps of $50 \mathrm{~Hz}$.
Once the region where the maximum frequency was identified, smaller steps of $10 \mathrm{~Hz}$ were used to more accurately identify the peak frequency and the actual resonant frequency, $\mathrm{f}_{\mathrm{n}}$.

$$
E=m\left(2 \pi f_{n}\right)^{2}\left(\frac{L}{A}\right)
$$

The modulus from vibrational studies was determined using equation (1) where $\mathrm{m}, \mathrm{L}$ and A are the sample mass, length and crosssectional area.

In Vivo determination of the resonant frequency of human skin and scar: In vivo studies on the mechanical properties of skin and healed scar tissue were conducted by hard wiring a $24 \mathrm{~mm}$ x $14 \mathrm{~mm}$ rectangular speaker (Digi-Key, Thief River Falls, MN) to a Samsung cell phone. A frequency generating app was downloaded from the Google Play Store onto the cell phone. This app was capable of driving the speaker between 10 and $20,000 \mathrm{~Hz}$. The speaker was taped to skin on a hand using surgical tape and it was used to generate a sinusoidal sound wave that vibrated the skin. During in vivo measurements, no sensation of the light or sound impinging on the skin was felt. The sound intensity was low enough so that it could not be detected unless the speaker was placed near the subject's ear to make sure it was energized.

The Digi-Key speaker was used for in vivo measurements in place of the Intervox speaker described for the in vitro studies. ${ }^{5,6}$ The speaker was located about $2.5 \mathrm{~cm}$ from the where the incident light beam contacted the skin and did not interfere with impingement of the light on the skin. The location of the incident beam on the skin influenced the extent of the displacement but not the resonant 
frequency measured as discussed previously. ${ }^{5,6,12}$ The optical signal generated by vibrating the skin with the Digi-Key speaker was then processed in the same manner as was done for in vitro studies and the resonant frequency was obtained by determination of the frequency at which the displacement was maximized.

For elastic modulus computation, the density of the normal skin and scar tissue was assumed to be same as that of decellularized dermis samples from previous studies. ${ }^{5,6}$ Based on the previously performed calibration study $y^{6,12}$ and equation (1), an equation derived from the relationship between the material density, resonant frequency, and elastic modulus of the material was used to compute the in vivo moduli for normal skin and scar tissue. The results of studies relating strain and modulus of decellularized dermis ${ }^{6,12}$ from used to back calculate the strain of normal skin and scar tissue in vivo.

\section{Calibration studies}

The relationship between the modulus measured using vibrational and tensile measurements was reported previously to be approximately linear and the equation of the line was found to be:

$$
\mathrm{Ev}=1.026 \mathrm{Et}+0.0046
$$

Where, Ev and Et are the moduli measured using vibrational and tensile measurements, respectively and are in MP as. ${ }^{6,12}$ The relationship between tensile and vibrational moduli was approximated using equation (2). The material behavior was reported to be reversible for strains less than about $14 \%$ for up to three cycles of tensile testing. ${ }^{5,6}$

Measurements made using the Digi-Key speaker in vitro were correlated with measurements made using the Intervox speaker in vitro by testing the same material using both speaker systems as previously reported. ${ }^{6}, 12$

\section{Results}

The relationship between moduli determined from vibrational and tensile experiments has been reported earlier. ${ }^{5,6,12}$ Using the vibrational method, the modulus of a material at a specific strain was calculated.

The resonant frequency and moduli were determined for human skin and scar, pig skin, intact bovine cartilage, subchondral bone and decellularized human dermis as shown in Table 1. Plots of weighted displacement versus frequency are shown for decellularized human dermis (Figure 1), pig skin (Figure 2), human skin and scar (Figure 3 ), intact bovine femoral cartilage (Figure 4) and subchondral bovine bone (Figure 5). The resonant frequency varied from about $70 \mathrm{~Hz}$ for human skin in vivo to over $600 \mathrm{~Hz}$ for subchondral bone (Table 1). The modulus determined from vibrational measurements varied from about $0.66 \mathrm{MPa}$ for human skin to over $30 \mathrm{MPa}$ for subchondral bone. Figure 1 illustrates that the resonant frequency of decellularized human dermis studied in vitro increases with increasing strain. Figure 2 illustrates that pigskin demonstrates two resonant frequencies one at $90 \mathrm{~Hz}$ and another at $200 \mathrm{~Hz}$. Figure 3 demonstrates that human skin studied in vivo shows a resonant frequency of $70 \mathrm{~Hz}$ with a small peak at about $180 \mathrm{~Hz}$ while scar tissue has a resonant frequency at $220 \mathrm{~Hz}$ with a small peak at about $70 \mathrm{~Hz}$. Finally, bovine femoral cartilage shows three resonant frequencies: one at $250 \mathrm{~Hz}$, one at $550 \mathrm{~Hz}$, and the final one at $660 \mathrm{~Hz}$. After the cartilage is removed from the subchondral bone with a scalpel, the resonant frequency of subchondral bone becomes $620 \mathrm{~Hz}$.

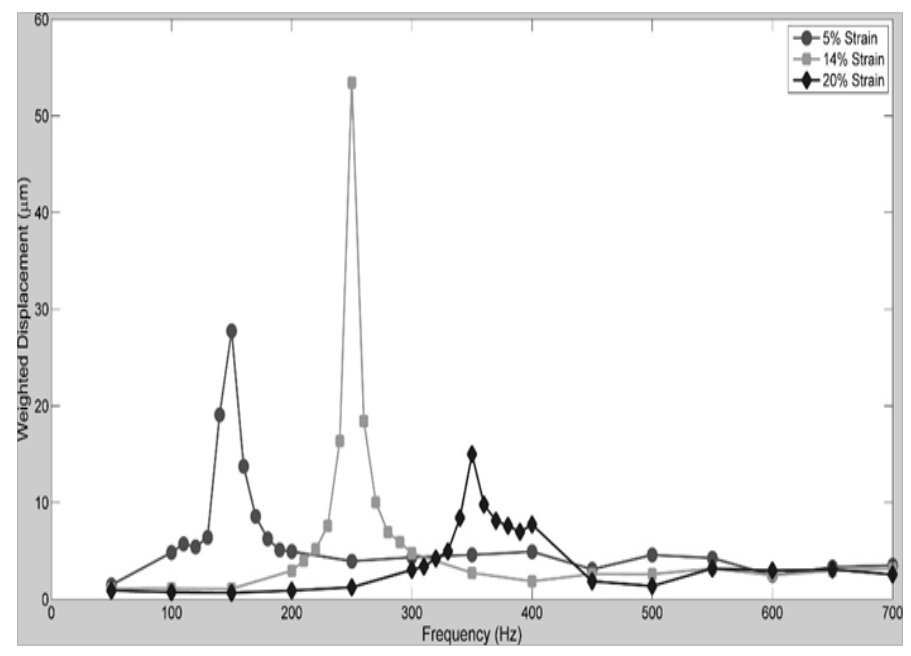

Figure I Weighted displacement versus frequency for decellularized dermis at $5 \%, 14 \%$ and $20 \%$ strain. The resonant frequencies measured were $150 \mathrm{~Hz}$ (5\% strain), $250 \mathrm{~Hz}$ ( $14 \%$ strain), and $350 \mathrm{~Hz}$ (20\% strain) as listed in Table I.

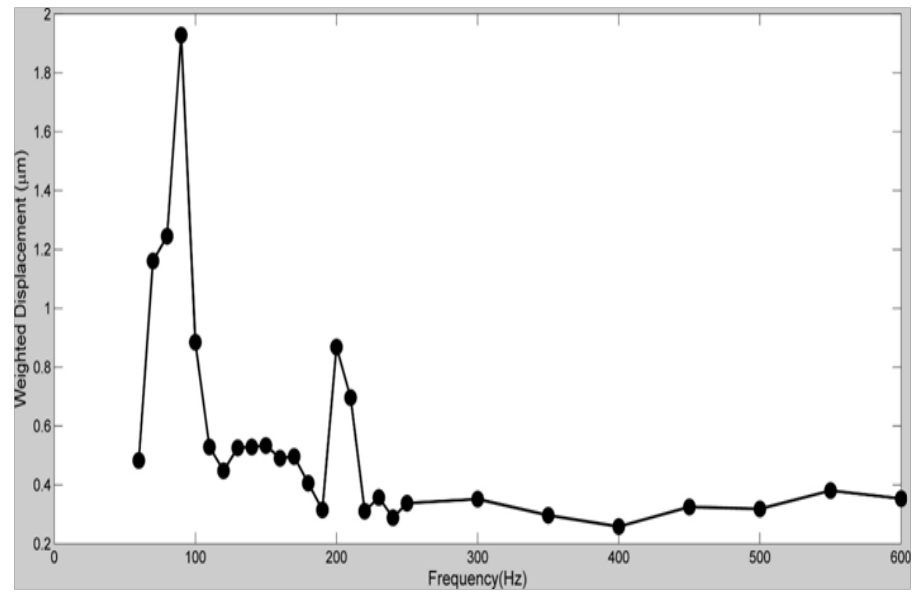

Figure $\mathbf{2}$ Weighted displacement versus frequency for pigskin stretched to a strain of $5 \%$. The resonant frequencies of the pigskin were $90 \mathrm{~Hz}$ (elastic fibers) and $200 \mathrm{~Hz}$ (collagen fibers) see Table I.

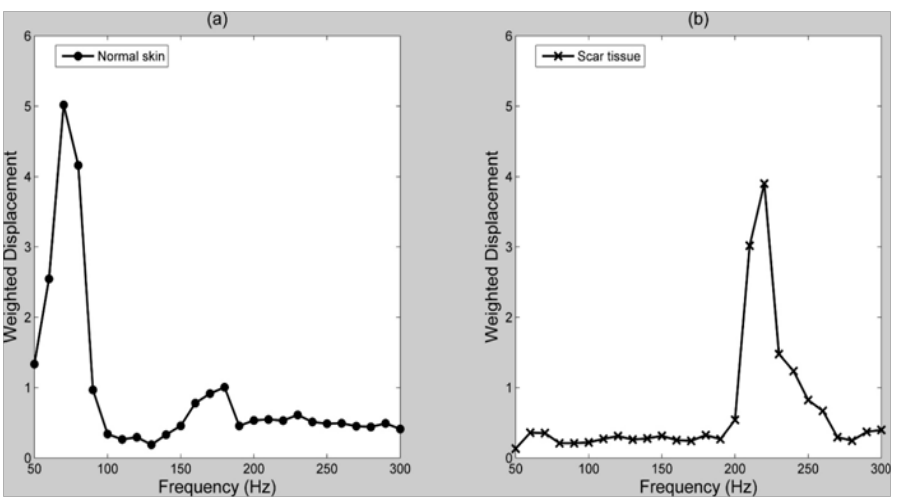

Figure 3 Weighted displacement versus frequency for normal skin (left) and scar tissue (right). The resonant frequencies were $70 \mathrm{~Hz}$ (normal skin) and 220 (scar tissue) as is listed in Table I. Note the small peaks in normal skin at about $180 \mathrm{~Hz}$ (postulated to be collagen fibers) and in scar at $70 \mathrm{~Hz}$ (postulated to be elastic fibers). 


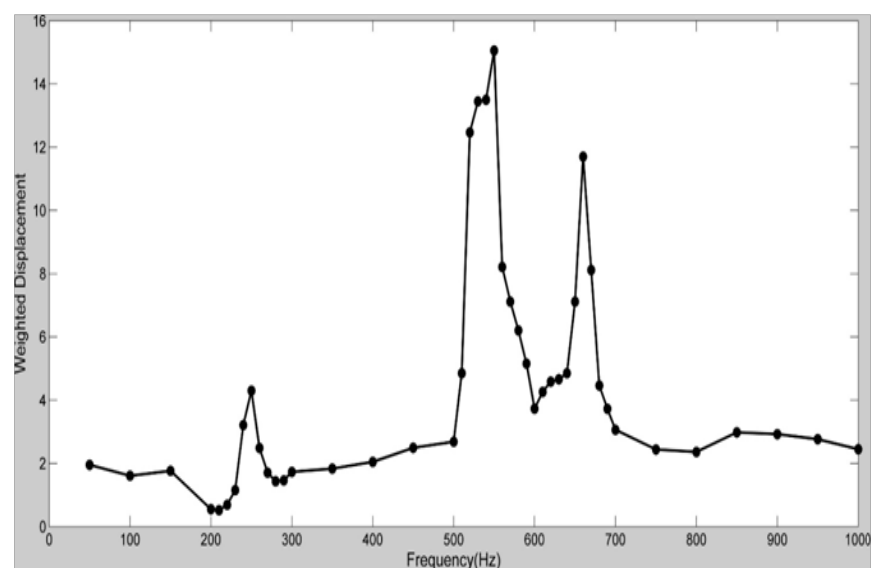

Figure 4 Weighted displacement versus frequency for bovine femoral cartilage with a layer of subchondral bone.The resonant frequencies observed were $250 \mathrm{~Hz}$ (postulated to be collagen fibers), $550 \mathrm{~Hz}$ and $660 \mathrm{~Hz}$ (postulated to be subchondral bone) as is listed in Table I.

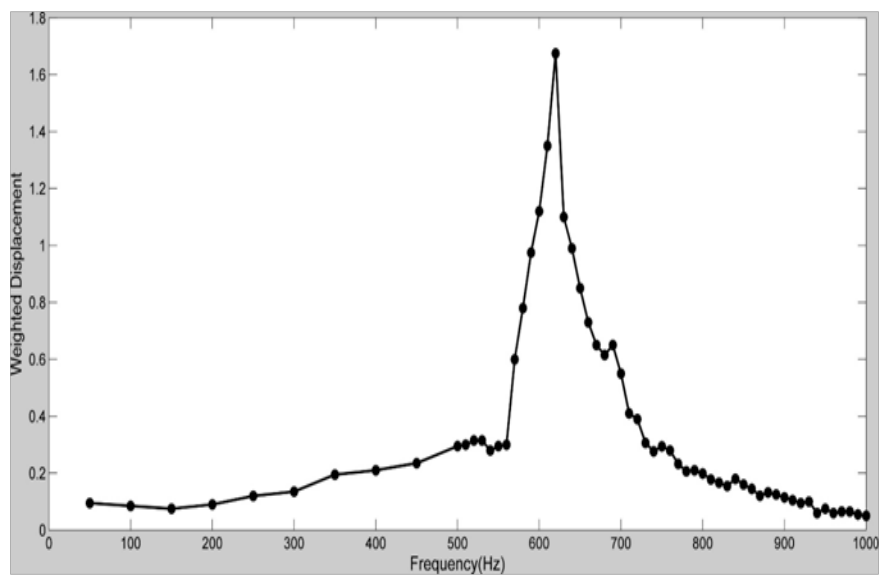

Figure 5 Weighted displacement versus frequency for bovine femoral cartilage and subchondral bone after the cartilage was removed with a scalpel. The resonant frequency was $620 \mathrm{~Hz}$ as is listed in Table I.

\section{Discussion}

The ability of researchers and clinicians to understand the relationship between ECM hierarchical structure and mechanical properties is an important issue in designing new tissue engineering scaffolds that mimic the structure and mechanical properties of the tissues they are replacing. While the mechanical properties of ECMs are complex, much progress has been made in understanding the relationship between collagen fiber organization and mechanical properties.

We recently reported a correlation between modulus values calculated from the slopes of incremental stress-strain curves and measurements based on natural frequency determination. ${ }^{5,6}$ These results suggest that the modulus determined from conventional methods (incremental stress-strain measurements) is consistent with the modulus values calculated from measurement of the natural frequency determined from a combination of OCT and vibrational analysis. ${ }^{5,6,12}$ The use of equation (1) to construct a calibration curve between modulus and natural frequency measurements results in a high correlation coefficient between these two independent measurements without assuming a value of Poisson's ratio. This suggests that use of vibrational analysis in conjunction with OCT gives modulus values that are similar to those found from uniaxial tensile measurements, a "gold standard" method that has been used to report stress-strain behavior of skin. ${ }^{1-4}$

One advantage of using the vibrational method is that the test is non-destructive and can be used to measure the mechanical properties of scar tissue in vivo. Using vibrational analysis, both natural and synthetic implant materials can be characterized non-invasively prior to, during, and after implantation.

One problem associated with characterization of these scaffolds, is to identify each peak in the vibrational spectrum. This task is simplified by analyzing the behavior of decellularized human dermis since it contains a single component, e.g. collagen fibers. ${ }^{9}$ The modulus of collagen fibers in skin increases from about $2.45 \mathrm{MPa}$ ( $5 \%$ strain) to $13.36 \mathrm{MPa}$ at a strain of $20 \%$ (Table 1 ). The resonant frequency and calculated modulus are therefore dependent on the tissue strain and the composition. If we next examine the weighted displacement curve versus frequency for pig skin we see two peaks; one at $90 \mathrm{~Hz}$ and another at $200 \mathrm{~Hz}$ (Figure 2). The peak at $200 \mathrm{~Hz}$ appears to be the collagen peak since it has a modulus of about $3.55 \mathrm{MPa}$ as opposed to the one for collagen in decellularized dermis with a modulus of $2.45 \mathrm{MPa}$. While the peak at $90 \mathrm{~Hz}$ is probably a peak due to elastic tissue since it has a modulus of 0.75 very close to that reported for elastic tissue in previous publications. ${ }^{13}$ In the case of human skin in vivo, the peak at $70 \mathrm{~Hz}$ with a modulus of $0.66 \mathrm{MPa}$ is probably a fingerprint for elastic tissue in the skin; whereas the peak at $220 \mathrm{~Hz}$ for scar tissue probably derives from the collagen fibers, since it has a modulus of $4.2 \mathrm{MPa}$.

One might next ask why the collagen peak in the normal skin measurements made in vivo is just a small hump in the curve (Figure $3,180 \mathrm{~Hz}$ peak). At low strains in vivo skin, the collagen fibers are stretched minimally in tension, whereas the elastic fibers have been reported to bear the loads. ${ }^{8}$ In contrast, in scar tissue the collagen fibers bear all the tension, even at low strains since there is only a small elastic fiber peak in scar tissue (Figure 3 small peak at $70 \mathrm{~Hz}$ ). This is consistent with a previous observation that human hypertrophic scar tissue has the same stiffness as normal human skin at high strains even though it appears more rigid by palpitation. ${ }^{14}$ The apparent increased stiffness of hypertrophic scar tissue is due to a reduced extensibility of this tissue. ${ }^{14}$ Since the elastic fibers are not replaced after thermal injury, elastic fibers do not contribute significantly to the modulus.

The data obtained with bovine femoral cartilage suggests that the peak at $250 \mathrm{~Hz}$ may reflect the collagen fibers in the superficial and intermediate zones. They have a modulus of $5.09 \mathrm{MPa}$, while the peak at about $600 \mathrm{~Hz}$ reflects the behavior of the subchondral bone on which the cartilage sits since it has a modulus of over $30 \mathrm{MPa}$ (Figure 5). The peak at 550 may reflect the transition zone between calcified cartilage and the subchondral bone.

The ability to measure the resonant frequency and modulus of normal and pathologic tissues using OCT and vibrational analysis may allow clinicians and scientists to better study and diagnose disease processes in both laboratory animals and in humans. In addition, it will assist workersto design better tissue engineered implants and scaffolds. The ability to follow changes in the mechanical properties of the components of skin implants and tissue engineered materials during healing and resorption would be an added advantage in minimizing 
the number of animals sacrificed in pre-clinical evaluations. The fact that the light beam used in these studies is as small as $14 \mu \mathrm{m}$ allows for localized analysis of tissue properties and tissue-implant interfaces. This may prove useful in analyzing healing at the implant-tissue interface as well as interfacial boundaries at the margins of wound tissue, tumors, ulcers, and scars in animal and human studies.

Since many ECMs such as skin are under tension in vivo, the strain in vivo is not the same as the strain measured in in vitro experiments. Typically, the strain in vivo is larger than that strain in in vitro experiments at the same external deformation due to the pre-stress that exists in tissue in vivo. To correct for this difference, age and location matched skin is tested in vitro to calibrate the in vivo measurements.

\section{Conclusion}

Our studies suggest that the mechanical properties of the macromolecular components of skin, scar, cartilage and subchondral bone can be obtained using a technique involving OCT and vibrational analysis. The high correlation between moduli measured using vibrational studies and uniaxial tensile tests suggest that the modulus can be determined by measuring the natural frequency that occurs when a tissue is vibrated in tension.

The results of studies on ECMs suggest that changes in mechanical behavior of the components of these tissues can be assessed noninvasively and non-destructively in vivo opening up new areas of research for analyzing non-invasively tissue repair and regeneration.

\section{Acknowledgements}

None.

\section{Conflict of interest}

The author declares no conflict of interest.

\section{References}

1. Silver FH. Biomaterials, Medical Devices and Tissue Engineering: An Integrated Approach. London, UK: Chapman and Hall; 1994. 303 p.

2. Silver FH, Shah R. Measurement of mechanical properties of natural and engineered implants. Advances in Tissue Engineering and Regenerative Medicine. 2016;1(1):1-9.
3. Yamada H. Strength of Biological Materials. Baltimore, Maryland, USA: Williams and Wilkins; 1970.

4. Fung YC. Biomechanics: Mechanical Properties of Living Tissue. 2nd ed. New York, USA: Springer; 1981. 568 p.

5. Silver FH, Shah RG. Viscoelastic behavior of allografts and scaffolds composed of extracellular matrices. Advances in Tissue Engineering and Regenerative Medicine. 2017;2(1):1-6.

6. Shah RG, Pierce MC, Silver FH. Morphomechanics of dermis-A method for non-destructive testing of collagenous tissues. Skin Res Technol. 2016;23(3):399-406.

7. Shah R, Pierce MC, Silver FH. A method for non-destructive mechanical testing of tissues and implants. J Biomed Mater Res A. 2017;105(1):522.

8. Dunn MG, Silver FH. Viscoelastic behavior of human connective tissues: Relative contribution of viscous and elastic components. Connect Tissue Res. 1983;12(1):59-70.

9. Silver FH, Freeman JW, DeVore D. Viscoelastic properties of human skin and processed dermis. Skin Res Technol. 2001;7(1):18-23.

10. Seehra GP, Silver FH. Viscoelastic properties of acid- and alkaline-treated human dermis: A correlation between total surface charge and elastic modulus. Skin Res Technol. 2006;12(3):190-198.

11. Shah RG, Silver FH. Viscoelastic behavior of tissues and implant materials: Estimation of the elastic and viscous contribution using optical coherence tomography and vibrational analysis. $J$ Biomed Tech Res. 2017;3(1):105

12. Shah RG, DeVore D, Silver FH. Biomechanical analysis of decellularized dermis and skin: Initial in vivo observations using OCT and vibrational analysis. J Biomed Mater Res A. 2018; doi: 10.1002/jbm.a.36344.

13. Shah RG, DeVore D, Pierce M, et al. Vibrational analysis of implants and tissues: Calibration and mechanical spectroscopy of multi-component tissues. J Biomed Mat Res A. 2017;105(6):1666-1671.

14. Dunn MG, Silver FH, Swann DA. Mechanical analysis of hypertrophic scar tissue: Structural basis for apparent increased rigidity. $J$ Invest Dermatol. 1985;84(1):9-13. 